\title{
Reconnaissance des unités fonctionnelles «salle d'opération de cabinet médical», «salle d'opération I» dans les cabinets médicaux de médecins praticiens
}

Service tarifaire de la FMH

En relation avec le recensement de la valeur intrinsèque de la FMH et le maintien des droits acquis afférents, des questions ont été soulevées quant aux délais provisoires accordés aux salles d'opération en cabinet médical. L'article présente le mode de procédure de reconnaissance et quels sont les délais à respecter.

\section{Définition des unités fonctionnelles «salle d'opération de cabinet médical», «salle d'opération I»}

De nombreuses demandes de renseignements le montrent, la définition de la salle d'opération dans les cabinets médicaux n'est pas des plus évidentes. Dans le cabinet du médecin de premier recours, la salle où se pratiquent des interventions est le plus souvent désignée sous la formule «salle d'opération de cabinet médical». Mais pour le TARMED, ce secteur est désigné sous le terme de «salle d'examen et de traitement, chirurgie». Selon le TARMED, une salle d'opération de cabinet médical est un espace qui ressemble à la salle d'opération d'une clinique. Ce local ne saurait dès lors avoir la fonction annexe de salle de consultation, comme c'est le cas dans la plupart des cabinets de médecins de premier recours.

La «salle d'opération I», par contre, répond aux normes d'une salle d'opération d'hôpital. Elle est nettement plus grande que la «salle d'opération de cabinet médical». Nombre de cabinets médicaux, principalement dans les disciplines chirurgicales, ont des salles répondant aux normes d'une «salle d'opération I».

\section{Facturation selon le TARMED}

En ce qui concerne la facturation des prestations, nombre de positions ont été créées. En premier lieu, toutes les prestations TARMED soumises à l'unité fonctionnelle «salle d'opération I» peuvent être exécutées dans une «salle d'opération de cabinet médical». Pour la facturation «salle d'opération de cabinet médical», la taxe de base par séance est calculée selon le chiffre 35.0010. Pour toutes les prestations fournies au cours de cette séance, les composantes de la prestation technique (PT) doivent cependant être diminuées de 40\% (selon la position 35.0020).

$\mathrm{Si}$ les prestations sont exécutées dans une «salle d'opération I» reconnue, c'est la prestation technique de base «salle d'opération I» (35.0030) qui peut être facturée. Les composantes PT des différentes prestations peuvent être facturées sans déduction.

\section{Reconnaissance des «salles d'opération de cabinet médical» / dispositions transitoires}

L'ancien tarif bleu de la SUVA disposait déjà d'un tarif spécial pour les prestations qui étaient exécutées dans une «salle d'opération de cabinet médical» reconnue d'un cabinet médical. Ces prestations étaient majorées d'un supplément de 80\% au lieu de 30\%. Dans le régime du TARMED, ces prestations ne peuvent être facturées que si le cabinet est reconnu comme «salle d'opération de cabinet médical». Les cabinets médicaux qui ne disposent pas de reconnaissance pour ce type d'unité fonctionnelle, ne peuvent plus facturer les prestations du tarif TARMED selon le secteur «salle d'opération I».

Comme on pouvait s'y attendre, cette réglementation pose problème dans un nombre non négligeable de cabinets médicaux, un grand nombre de prestations ne pouvant plus être facturées. La reconnaissance de ces prestations ne demeure en effet possible que si elles satisfont aux exigences fixées par le TARMED. La FMH a néanmoins convenu avec les organismes payeurs que pour une durée transitoire d'une année, les salles d'opération de cabinets médicaux ne remplissant pas entièrement les critères 
exigés pouvaient encore être reconnues. La procédure de reconnaissance demeure la même. Les cabinets médicaux provisoirement reconnus reçoivent une liste de critères à remplir. Si ces lacunes sont comblées au bout d'une année, ils reçoivent une reconnaissance définitive. Les autres ne sont plus autorisés, à partir de ce moment, à facturer les prestations «salle d'opération de cabinet médical». Des exceptions demeurent dans les cantons qui disposent déjà de reconnaissances propres pour les cabinets médicaux avec «salle d'opération de cabinet médical» inhérents au domaine LAMal (selon entente entre les assureurs-maladie et les sociétés cantonales de médecine concernées). Ces cabinets avec «salle d'opération de cabinet médical» reçoivent pendant trois ans suivant l'introduction du TARMED les reconnaissances LAMal. Après trois ans, ces cabinets avec «salle d'opération de cabinet médical» doivent cependant remplir entièrement les conditions fixées par le TARMED.

Un programme d'assurance qualité est lié à la reconnaissance. Chaque année, un spécialiste de l'hygiène effectue un contrôle par échantillonnage de 5\% des unités fonctionnelles reconnues. Les coûts de ces contrôles sont répartis sur l'ensemble des cabinets médicaux concernés.

\section{Reconnaissance des «salles d'opération I" en cabinet médical}

Contrairement à ce que l'on pense souvent, il est parfaitement possible de gérer une «salle d'opération I» dans un cabinet médical. Il suffit de remplir les conditions nécessaires pour recevoir l'autorisation de facturer les prestations au moyen des positions tarifaires pour les «salles d'opération I». La procédure de reconnaissance est analogue à celle des "salle d'opération de cabinet médical» par l'intermédiaire du groupe de travail «chirurgie ambulatoire» PARODIG/ TARMED Suisse (secrétariat auprès du Service tarifaire de la FMH, fmhtarif@hin.ch). Le groupe de travail effectue le tri des demandes. Toutes les «salle d'opération de cabinet médical» sont répertoriées dans la banque de données des unités fonctionnelles de la FMH et toutes les «salles d'opération I» dans celle de H+. En ce qui concerne les reconnaissances, les médecins en pratique privée ne peuvent s'adresser qu'à la FMH. Ils ne sont pas autorisés à demander une reconnaissance directement par le programme $\mathrm{H}+$.

Celui qui dispose d'une «salle d'opération I» reconnue dans son cabinet médical demeure intégré dans les programmes de contrôle qualité des «salles d'opération de cabinet médical».

Tableau 1

Aperçu des conditions de reconnaissance des «salles d'opération de cabinet médical» et les «salles d'opération l» ainsi que des salles d'examen et de traitement dans les cabinets médicaux.

\begin{tabular}{|l|l|l|l}
\hline Définition & $\begin{array}{l}\text { Salle d'opération } \\
\text { de cabinet médical }\end{array}$ & Salle d'opération I & $\begin{array}{l}\text { Salle d'examen } \\
\text { et de traitement }\end{array}$ \\
\hline $\begin{array}{l}\text { Correspond à peu près } \\
\text { à la salle d'opération d'une } \\
\text { clinique. Ne saurait avoir } \\
\text { la fonction d'une salle } \\
\text { de consultation }\end{array}$ & $\begin{array}{l}\text { Répond aux normes d'une } \\
\text { salle d'opération d'hôpital; } \\
\text { nettement plus grande } \\
\text { qu'une «salle d'opération } \\
\text { de cabinet» }\end{array}$ & $\begin{array}{l}\text { Salle où se pratiquent } \\
\text { des interventions dans } \\
\text { le cabinet du médecin } \\
\text { de premier recours }\end{array}$ \\
\hline selon le TARMED & $\begin{array}{l}\text { GTaxe de base } 35.0010 \text { par } \\
\text { séance, mais les composantes } \\
\text { de la prestation technique } \\
\text { doivent être diminuées } \\
\text { de } 40 \% \text { (selon la position } \\
\text { 35.0020) pour toutes les } \\
\text { prestations fournies }\end{array}$ & $\begin{array}{l}\text { Prestation technique de base } \\
\text { «salle d'opération l» } \\
\text { (35.0030) par séance. } \\
\text { Les composantes de la } \\
\text { prestation technique peuvent } \\
\text { être facturées sans déduction } \\
\text { pour toutes les prestations }\end{array}$ & $\begin{array}{l}\text { Selon la position tarifaire } \\
\text { correspondante }\end{array}$ \\
\hline Reconnaissance & $\begin{array}{l}\text { Nécessaire pour facturer des prestations tarifiées selon l'unité } \\
\text { fonctionnelle «salle d'opération l». Les droits acquis ne sont pas } \\
\text { applicables aux unités fonctionnelles }\end{array}$ & Pas nécessaire \\
\hline $\begin{array}{l}\text { Dispositions } \\
\text { transitoires }\end{array}$ & \begin{tabular}{l} 
Délai d'adaptation d'une année \\
\hline Exceptions
\end{tabular} & $\begin{array}{l}\text { Existent dans certains cantons, selon entente entre } \\
\text { les assureurs-maladie et les sociétés cantonales de médecine } \\
\text { concernées }\end{array}$ & \\
\hline
\end{tabular}




\section{Normes pour les «salles d'opération de cabinet médical» et «les salles d'opération I»}

Les exigences liées aux unités fonctionnelles peuvent s'appuyer sur le concept de reconnaissance des secteurs TARMED. Le concept figure sur l'internet par le biais de l'adresse suivante: www.fmh.ch (Tarifs $\rightarrow$ Tarmed/AA/AM/AI $\rightarrow$ Convention concernant la reconnaissance des unités fonctionnelles). En cas de doutes concernant l'une ou l'autre exigence, le service tarifaire de la FMH donne volontiers des informations.

\section{Voie de recours}

En cas de décision négative du groupe de travail «chirurgie ambulatoire», un recours peut être interjeté par écrit dans les 30 jours auprès du groupe PAKODIG.

\section{Coûts de la reconnaissance}

Les coûts pour la reconnaissance se montent à Fr. 500.-. Ce montant permet de couvrir les frais des parties contractantes pour la vérification et les frais de secrétariat. Les frais de recours contre une décision du groupe de travail «chirurgie ambulatoire» se montent à Fr. 300.-, auxquels s'ajoutent les frais d'une visite sur place. Si le recours est accepté, ces coûts sont remboursés.

Les coûts des contrôles annuels de qualité s'élèvent à env. Fr. 200.-. Ils devraient cependant encore baisser, suivant les possibilités. 